\title{
Adverse Pregnancy Outcome among Different Maternal Age group - A Comparative Study
}

\author{
Dr. Serajoom Munira ${ }^{1 *}$, Professor Roksana Ivy ${ }^{2}$, Professor Fatema Ashraf ${ }^{3}$, Dr. Mohammad Ibrahim Khalil ${ }^{4}$ \\ ${ }^{1}$ Consultant, Department of Gynae \& Obs. Shaheed Suhrawardy Medical College \& Hospital, Dhaka Bangladesh \\ ${ }^{2}$ Professor Department of Gynae \& Obs, Shaheed Suhrawardy Medical College \& Hospital, Dhaka Bangladesh \\ ${ }^{3}$ Professor \& Head of Department, Gynae \& Obs. Shaheed Suhrawardy Medical College \& Hospital, Dhaka Bangladesh \\ ${ }^{4}$ Associate Professor Department of Neurology, Shaheed Suhrawardy Medical College \& Hospital, Dhaka Bangladesh
}

\begin{abstract}
DOI: $10.36348 /$ sijog.2020.v03i07.001 $\quad$ | Received: 31.05 .2020 | Accepted: 08.06.2020 | Published: 12.07 .2020
*Corresponding author: Dr. Serajoom Munira
\end{abstract}

\section{Abstract}

Background: Advanced maternal age generally signifies age after 35years during the time of delivery. Although the fact that being pregnant at any reproductive age is not risk-free, older gravidity usually caps with adverse outcomes both to the mother and fetus or neonate. Objective: The key objective of this study was to compare pregnancy outcomes associated with three different maternal age groups. Materials and Method: It was a comparative observational study performed at the Department of Obstetrics and Gynecology, Shaheed Suhrawardy Medical College \& Hospital, Dhaka, Bangladesh. The study included all women who delivered in gyane \& Obs department at 24 weeks' gestation period from January 2017 to December 2018. Results: In this study, three maternal groups were considered as the younger age group of 18-29 years, middle age group of 30-34 years, and advanced mother age (AMA) 35-40 years in maternal age. Chronic hypertension was significantly associated with maternal age. However, antepartum hemorrhage, placenta previa, abruptio placenta, gestational hypertension, preeclampsia, eclampsia, gestational diabetes mellitus (GDM), and bad obstetrics history $(\mathrm{BOH})$, were not statistically significant $(\mathrm{p}>0.05)$. Regarding malpresentation, breach, transverse lie, congenital malformation, intrauterine device (IUD), preterm labour, prolonged labour, obstructed labour and postpartum hemorrhage $(\mathrm{PPH})$ were not statistically significant $(\mathrm{p}>0.05)$. Low birth weight was found 38(30.9\%) in the age group 18-29 years, $9(20 \%)$ of 30-34 years, and 3(18.8\%) in the age group 35-40 years. Intrauterine growth restriction (IUGR) was found $3(2.4 \%)$, stillbirth $6(4.9 \%)$, prematurity $4(3.3 \%)$ and macrosoma $1(0.8 \%)$ in the age group 18-29 years, in the age group 35-40 years, NICU admission was needed 36(29.3\%) in the age group of 18-29 years,8(19.5\%) in age group30-34 years and 2(12.5\%) in the age group 35-40 years. The difference was not statistically significant ( $\mathrm{p}>0.05)$. Conclusion: In our findings demonstrate that though any reproductive age groups are not risk-free of pregnant women, over 35 years are at elevated risk for rising complications and should, therefore, be managed with proper care protocols, particularly in cases of maternal complications of pregnancy.

Keywords: Reproductive age, Advance maternal age, older gravidity, adverse outcomes.

Copyright @ 2020: This is an open-access article distributed under the terms of the Creative Commons Attribution license which permits unrestricted use, distribution, and reproduction in any medium for non-commercial use (NonCommercial, or CC-BY-NC) provided the original author and source are credited.

\section{INTRODUCTION}

An increasing number of women in developing countries are postponing pregnancy [1]. However, there's sparse data regarding very advanced maternal age (vAMA), and therefore the interplay between vAMA and assisted reprocessing prevalence of women who tend to delay childbirth until a very advanced age. Active technology (ART) on adverse perinatal outcomes [2]. The age at which women deliver their first child has increased gradually within the past few decades, predominantly in Western societies. Births occurring among women aged 35 years and older are increasing and constitute $14 \%$ and $18 \%$ of total live births within the USA and Canada, respectively [3]. But the birth rate in women of 20-24 age group declined from $80.7 \%$ in 2013 to $79.0 \%$ in 2014 , considering this rate was as high as $115.1 \%$ in 1980[4]. In Japan, the rate of birth to women aged 35 above elevated from $8.6 \%$ in 1990 to $25.9 \%$ in 2012.5 Similar trends are found in other developed countries[5-9]. The association of advanced maternal age (AMA) and 
increased risk of congenital abnormality, miscarriage, perinatal mortality, and maternal complications are reported in many studies in developed countries [5-9, 10]. Advanced maternal age may be a known risk factor for chromosomal abnormalities of the fetus and early complications of pregnancy like miscarriage and pregnancy [11-13]. Studies have reported an association between advanced maternal age and adverse pregnancy outcomes, including low birth weight, preterm birth, fetal death and stillbirth, preeclampsia, abruption, placenta praevia, and maternal death [14-16].

\section{MATERIALS AND METHOD}

It was a comparative observational study performed at the Department of Obstetrics and Gynecology, in a tertiary care Hospital. Dhaka, Bangladesh. The study included all women who delivered in our department at 24 weeks' gestation period from January 2017 to December 2018. The data were obtained from the hospital's computerized obstetric and neonatal database which includes information on maternal demographics, obstetric history, pregnancy and labor/delivery events, and shortterm maternal and neonatal outcomes. The validity and accuracy of the database are periodically controlled by a specific internal committee. Data included maternal age, pre-pregnancy body mass index (BMI, weight in kilograms divided by height in meters squared), parity, pre-existing chronic diseases (particularly chronic hypertension and diabetes), mode of conception, gestational age at delivery, complications of pregnancy, mode of delivery, post-partum complications, and neonatal outcome. Since many women over 40 years have twin pregnancy and/or ART pregnancies, women with twin pregnancies and ART pregnancies were included in the study. The primary outcomes were the rate of adverse pregnancy outcomes (maternal and/or perinatal) in women aged $\leq 35$ years and those over 35 years. Complications of pregnancy included gestational diabetes, late-onset (34 weeks' gestation), and early onset (33 to 36 weeks' gestation) preeclampsia, placenta praevia, abruption, and post-partum hemorrhage. Gestational diabetes was diagnosed in previously nondiabetic women according to American Diabetes Association criteria. According to the protocol of our department, gestational diabetes should be tested in all women over 35 years and in women less than 35 years with risk factors. Preeclampsia was diagnosed in previously normotensive and non-proteinuric women according to the American College of Obstetricians and gynecologists criteria. Perinatal outcomes included preterm birth either spontaneous or indicated (4000 g, < $2500 \mathrm{~g}$, and $<1500 \mathrm{~g}$, Apgar score $<7$ at $5 \mathrm{~min}$ ) major fetal anomalies, and perinatal death. Perinatal death included stillbirth, defined as the birth of a baby without any signs of life after 24 weeks' gestation, and neonatal death defined as death before 7 completed days after birth. The results are presented as frequencies and percentages or as mean \pm SD for categorical and continuous variables, respectively. For categorical variables, between-group comparisons were performed using chi-square. As appropriate. Univariate analysis was performed using ANOVA to compare the mean values of continuous variables between groups. Logistic regression models were performed for significantly different variables to assess the possible independent role of maternal age as a risk factor for adverse pregnancy outcomes. The results are presented showing odds ratios and $95 \%$ confidence interval $(\mathrm{CI})$ estimates. All statistical tests were two-sided. p- Values of $<0.05$ or less were considered statistically significant. Statistical analysis was conducted using the SPSS software package, version 23.0.

\section{RESULTS}

Table-I: Maternal characteristics in relation to maternal age $(n=180)$

\begin{tabular}{|c|c|c|c|c|c|c|c|}
\hline \multirow[t]{3}{*}{ Maternal characteristics } & \multicolumn{6}{|c|}{ Maternal age group } & \multirow[t]{3}{*}{$P$ value } \\
\hline & \multicolumn{2}{|c|}{$\begin{array}{c}\text { 18-29 years } \\
(\mathrm{n}=123)\end{array}$} & \multicolumn{2}{|c|}{$\begin{array}{c}\text { 30-34 years } \\
(\mathrm{n}=41)\end{array}$} & \multicolumn{2}{|c|}{$\begin{array}{c}35-40 \text { years } \\
(n=16)\end{array}$} & \\
\hline & $\mathrm{n}$ & $\%$ & $\mathrm{n}$ & $\%$ & $\mathrm{n}$ & $\%$ & \\
\hline Mean age (years) & \multicolumn{2}{|c|}{$22.8 \pm 3.1$} & \multicolumn{2}{|c|}{$30.9 \pm 1.2$} & \multicolumn{2}{|c|}{$35.4 \pm 0.9$} & $0.001^{\mathrm{s}}$ \\
\hline \multicolumn{8}{|l|}{ Parity } \\
\hline Primiparous & 59 & 48.0 & 15 & 36.6 & 3 & 18.8 & \multirow[t]{2}{*}{$0.056^{\mathrm{ns}}$} \\
\hline Multiparous & 64 & 52.0 & 26 & 63.4 & 13 & 81.3 & \\
\hline \multicolumn{8}{|l|}{ Antenatal check up } \\
\hline Regular & 51 & 41.5 & 13 & 31.7 & 8 & 50.0 & \\
\hline Irregular & 57 & 46.3 & 24 & 58.5 & 7 & 43.8 & $0.602^{\mathrm{ns}}$ \\
\hline No check up & 15 & 12.2 & 4 & 9.8 & 1 & 6.3 & \\
\hline \multicolumn{8}{|l|}{ Immunization } \\
\hline Yes & 77 & 62.6 & 29 & 7.1 & 9 & 56.3 & \multirow[t]{2}{*}{$0.515^{\mathrm{ns}}$} \\
\hline No & 46 & 37.4 & 12 & 2.9 & 7 & 43.8 & \\
\hline Mean gestational age (weeks) & \multicolumn{2}{|c|}{$38.05 \pm 2.37$} & \multicolumn{2}{|c|}{$37.43 \pm 3.09$} & \multicolumn{2}{|c|}{$37.66 \pm 2.25$} & $0.378^{\mathrm{ns}}$ \\
\hline Antepartum hemorrhage & 2 & 1.6 & 3 & 7.3 & 0 & 0.0 & $0.123^{\mathrm{ns}}$ \\
\hline Placenta previa & 0 & 0.0 & 2 & 4.9 & 0 & 0.0 & $0.032^{\mathrm{s}}$ \\
\hline Abruptio placenta & 0 & 0.0 & 1 & 2.4 & 0 & 0.0 & $0.181^{\mathrm{ns}}$ \\
\hline
\end{tabular}


Serajoom Munira et al; Sch Int J Obstet Gynec, July. 2020; 3(7): 163-169

\begin{tabular}{|l|c|c|c|c|c|c|c|}
\hline Gestational HTN & 6 & 4.9 & 7 & 17.1 & 3 & 18.8 & $0.020^{\mathrm{s}}$ \\
\hline Preeclampsia & 9 & 7.3 & 0 & 0.0 & 2 & 12.5 & $0.127^{\mathrm{ns}}$ \\
\hline Eclampsia & 3 & 2.4 & 0 & 0.0 & 0 & 0.0 & $0.493^{\mathrm{ns}}$ \\
\hline GDM & 5 & 4.1 & 2 & 4.9 & 2 & 12.5 & $0.346^{\mathrm{ns}}$ \\
\hline Chronic HTN & 0 & 0.0 & 0 & 0.0 & 1 & 6.3 & $0.005^{\mathrm{s}}$ \\
\hline BOH & 1 & 0.8 & 1 & 2.4 & 0 & 0.0 & $0.631^{\mathrm{ns}}$ \\
\hline Malpresentation & & & & & & & \\
\hline Breach & 6 & 4.9 & 4 & 9.8 & 2 & 12.5 & $0.343^{\mathrm{ns}}$ \\
\hline Transverse lie & 0 & 0.0 & 1 & 0.2 & 0 & 0.0 & $0.181^{\mathrm{ns}}$ \\
\hline Congenital malformation & 3 & 2.4 & 0 & 0.0 & 0 & 0.0 & $0.493^{\mathrm{ns}}$ \\
\hline IUD & 6 & 4.9 & 2 & 4.9 & 0 & 0.0 & 0.664 \\
\hline Preterm labour & 7 & 5.7 & 4 & 9.8 & 0 & 0.0 & $0.362^{\mathrm{ns}}$ \\
\hline Prolonged labour & 9 & 7.3 & 1 & 2.4 & 1 & 6.3 & $0.528^{\mathrm{ns}}$ \\
\hline Obstructed labour & 19 & 15.4 & 2 & 4.9 & 3 & 18.8 & $0.181^{\mathrm{ns}}$ \\
\hline PPH & 17 & 13.8 & 4 & 9.8 & 1 & 6.3 & $0.589^{\mathrm{ns}}$ \\
\hline Mode of delivery & & & & & & & \\
\hline \multicolumn{1}{|c|}{ Normal } & 52 & 42.3 & 16 & 39.0 & 3 & 18.8 & $0.193^{\mathrm{ns}}$ \\
\cline { 1 - 6 } C/S & 71 & 57.7 & 25 & 61.0 & 13 & 81.3 & \\
\hline
\end{tabular}

$\mathrm{s}=$ significant; $\mathrm{ns}=$ not significant

$P$ value reached from unpaired t-test and Chi square test

Table I shows the mean age was found $22.8 \pm 3.1$ years in the age group 18-29 years, $30.9 \pm 1.2$ years in the age group 30-34 years, and $35.4 \pm 0.89$ years in the age group 35-40 years. The difference was statistically significant $(p<0.05)$ between the three groups. Majority patients were multiparous in all three age groups, which were $64(52 \%)$ in the age group 1829 years, 26(63.4\%) in the age group 30-34 years and $13(81.3 \%)$ in the age group $35-40$ years. The difference was not statistically significant between parity and maternal age group. Regarding antenatal checkup, irregular antenatal checkup was found $57(46.3 \%)$ in the age group 18-29 years, $24(58.5 \%)$ in the age group 30 34 years, and $7(43.8 \%$ ) in the age group 35-40 years with. The difference was not statistically significant between antenatal check and maternal age group. Immunization was found $77(62.6 \%)$ in the age group $18-29$ years, $29(7.1 \%)$ in the age group 30-34 years, and $9(56.3 \%)$ in the age group 35-40 years respectively The difference was not statistically significant between immunization and maternal age group. Mean gestational age was found $38.05 \pm 2.37$ weeks in the age group 18-29 years, $37.43 \pm 3.09$ in age group, and $37.66 \pm 2.25$ weeks in the age group $35-40$ years. The difference was statistically significant $(p<0.05)$ between the three groups. Most of the patients had gestational hypertension $6(4.9 \%$ ) in the age group 18-29 years, $7(17.1 \%)$ in the age group $30-34$ years, and $3(18.8 \%)$ in age group 35-4 years. The difference was not statistically significant between gestational hypertension and maternal age groups. Preeclampsia was $9(7.3 \%)$ and $2(12.5 \%)$ in age group 18-29 and 3540 years respectively. Chronic hypertension was $1(6.3 \%)$ in age group 35-4 years. Chronic hypertension was significantly associated with maternal age. However, antepartum hemorrhage, placenta previa, abruptio placenta, gestational hypertension, preeclampsia, eclampsia, GDM, and $\mathrm{BOH}$ were not statistically significant $\quad(\mathrm{p}>0.05)$ Regarding malpresentation, breach, transverse lie, congenital malformation, IUD, preterm labour, prolonged labour, obstructed labour and PPH were not statistically significant $(\mathrm{p}>0.05)$. In the mode of delivery, normal delivery was found $52(42.3 \%$ ) in the age group 18-29 years, $16(39 \%)$ in the age group 30-34 years and $3(18.8 \%)$ in age group 35-40 years. Caesarean section was $71(57.7 \%)$ in the age group $18-29$ years, $25(16 \%)$ in the age group $30-34$ years, and $13(81.3 \%$ ) in the age group 35-4 years respectively. The difference was not statistically significant $(p>0.05)$ between mode of delivery and maternal age.

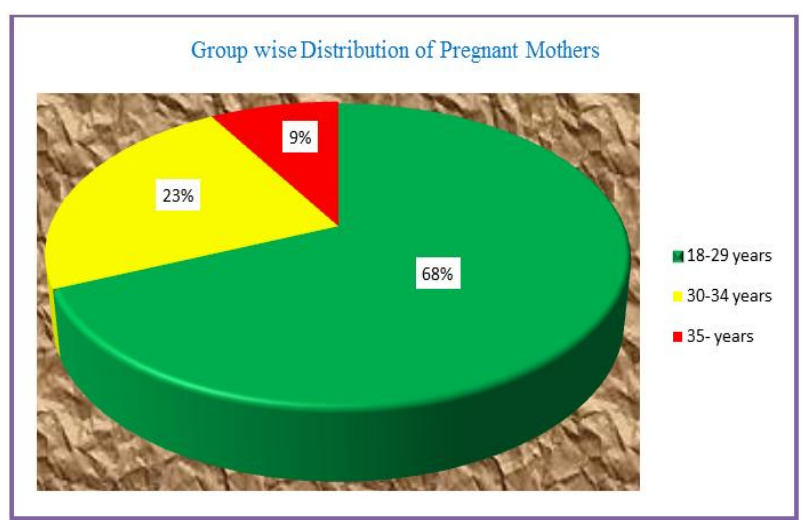

Fig-1: Group wise Distribution of Pregnant Mothers 


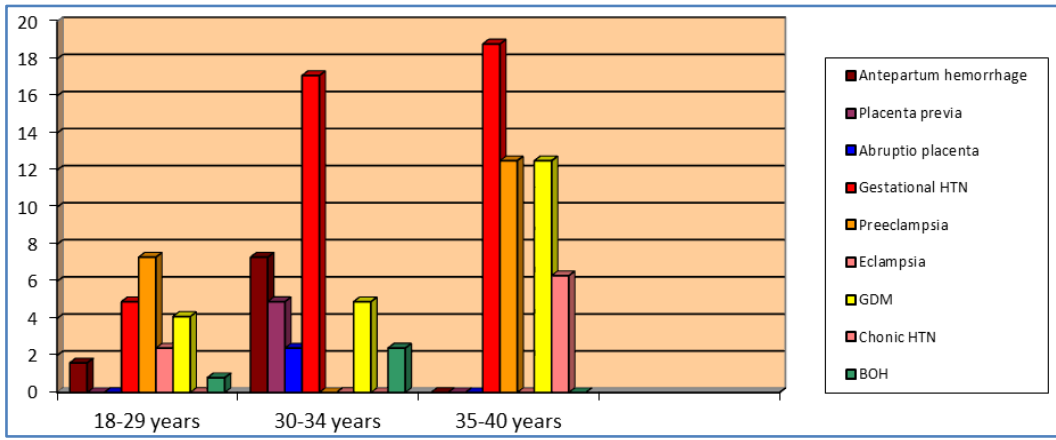

Fig-2: Distribution of Maternal Key Complications

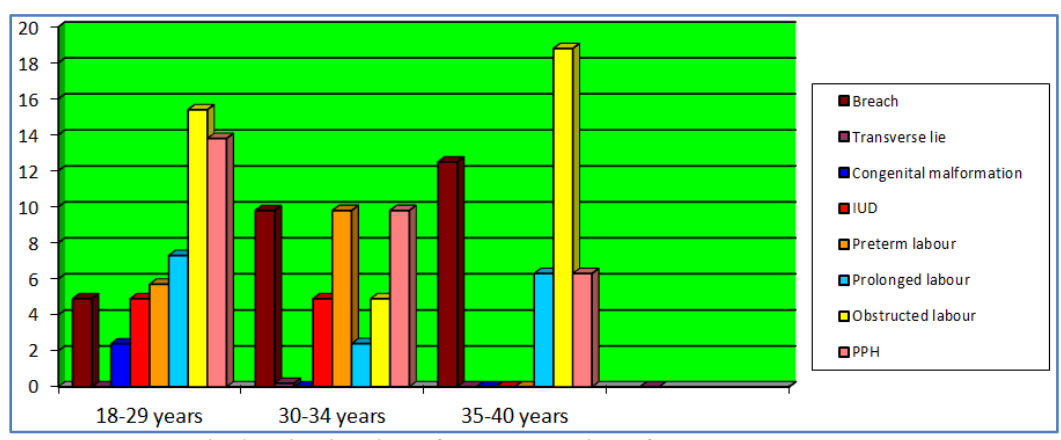

Fig-3: Distribution of Malpresention of Pregnant Mothers

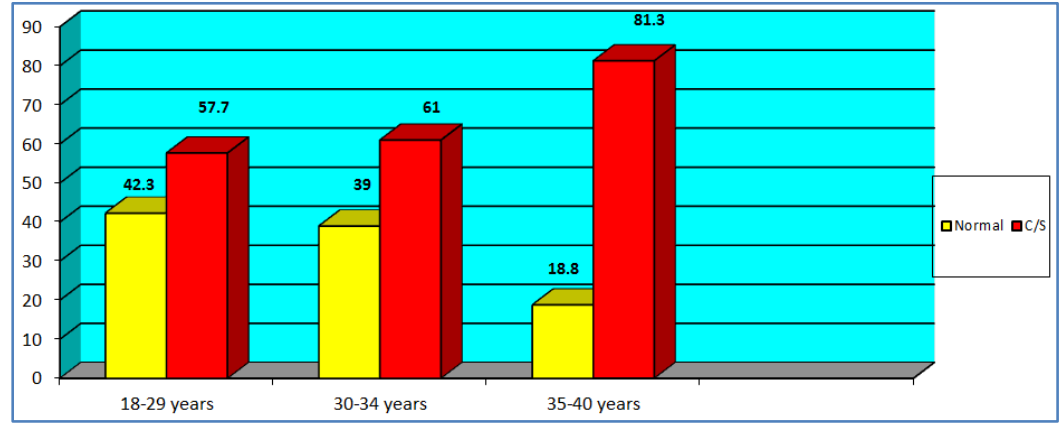

Fig-4: Mode of Delivery of Pregnant Mothers

Table-II: Fetal outcomes in relation to maternal age $(n=180)$

\begin{tabular}{|c|c|c|c|c|c|c|c|}
\hline \multirow[t]{3}{*}{ Fetal outcomes } & \multicolumn{6}{|c|}{ Maternal age group } & \multirow{3}{*}{$\begin{array}{c}\mathbf{P} \\
\text { value }\end{array}$} \\
\hline & \multicolumn{2}{|c|}{$\begin{array}{c}\text { 18-29 years } \\
(n=123)\end{array}$} & \multicolumn{2}{|c|}{$\begin{array}{c}30-34 \text { years } \\
(n=41)\end{array}$} & \multicolumn{2}{|c|}{$\begin{array}{c}35-40 \text { years } \\
(n=16)\end{array}$} & \\
\hline & $\mathrm{n}$ & $\%$ & $\mathrm{n}$ & $\%$ & $\mathrm{n}$ & $\%$ & \\
\hline Low birth weight & 38 & 30.9 & 9 & 22.0 & 3 & 18.8 & $0.379^{\mathrm{ns}}$ \\
\hline IUGR & 3 & 2.4 & 1 & 2.4 & 0 & 0.0 & $0.819^{\mathrm{ns}}$ \\
\hline Still birth & 6 & 4.9 & 2 & 4.9 & 0 & 0.0 & $0.664^{\mathrm{ns}}$ \\
\hline Prematurity & 4 & 3.3 & 3 & 7.3 & 0 & 0.0 & $0.355^{\mathrm{ns}}$ \\
\hline Macrosoma & 1 & 0.8 & 1 & 2.4 & 0 & 0.0 & $0.625^{\mathrm{ns}}$ \\
\hline NICU admission & 36 & 29.3 & 8 & 19.5 & 2 & 12.5 & $0.211^{\mathrm{ns}}$ \\
\hline
\end{tabular}

Table II shows low birth weight was found $38(30.9 \%)$ in the age group $18-29$ years, $9(22 \%)$ in the age group 30-34 years and 3(18.8\%) in age group 35-4 years. IUGR was found $3(2.4 \%)$, stillbirth $6(4.9 \%)$, prematurity $4(3.3 \%)$ and macrosoma $1(0.8 \%)$ in the age group 18-29 years on the other hand due to limited sample size only low birth weight was found $3(18.8 \%)$ in the age group 35-40 years. NICU admission was needed $36(29.3 \%)$ in the age group of 18-29 years, $8(19.5 \%)$ in the age group $30-34$ years and $2(12.5 \%)$ in the age group 35-40 years. The difference was not statistically significant $(\mathrm{p}>0.05)$. 


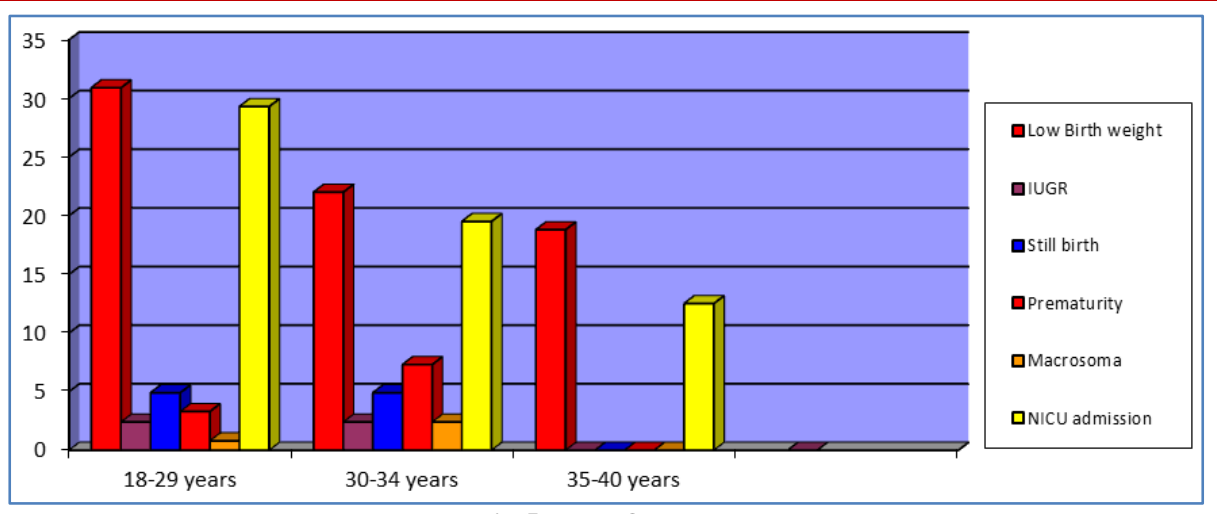

Fig-5: Fetal Outcomes

\section{DISCUSSION}

This study observed that the mean age was found 38.05 \pm 2.37 years in age 18-29 years, 37.43 \pm 3.09 in the age group 30-34 years, and $37.66 \pm 2.25$ years in age 35-40 years. The difference was statistically significant $(\mathrm{p}<0.05)$ between the three groups. A similar study showed that the mean maternal age was found $28.5 \pm 3.59$ in the age group of $20-34$ and $36.5 \pm 1.35$ in the age group of 35-39 years [17]. In this study the majority patients were multiparous in three age groups, which was $64(52 \%)$ in the age group 18-29 years, $26(63.4 \%)$ in the age group $30-34$ years and $13(81.3 \%)$ in the age group 35-40 years. The difference was not statistically significant between parity and maternal age group, the associations between maternal age and birth outcomes stratified dichotomously by maternal parity and conception method [18]. The effect of advanced age on an increased risk of cesarean section and emergency cesarean section was significantly greater among primiparous women than among multiparous women, observed that prim parity increased the selective CS risks with increasing maternal age, especially in AMA groups [17]. However, for emergency CS, there was a reduced risk in primiparous patients aged 40 years and above. As an aggravating factor for GDM (lifestyle intervention), primiparity increased this risk in patients of group 3, while it had no effect on older or younger counterparts. Regarding antenatal checkup, irregular antenatal checkup was found $57(46.3 \%$ ) in the age group 18-29 years, $24(58.5 \%)$ in the age group $30-34$ years, and $7(43.8 \%)$ in the age group 35-40 years. The difference was not statistically significant between antenatal check and maternal age group. Immunization was found $77(62.6 \%)$ in the age group $18-29$ years, $29(7.1 \%)$ in the age group $30-34$ years, and $9(56.3 \%)$ in the age group 35-40 years respectively. The difference was not statistically significant between immunization and maternal age group. Most of the patients had gestational hypertension $6(4.9 \%)$ in the age group 18-29 years, $7(17.1 \%)$ in the age group $30-34$ years, and $3(18.8 \%)$ in the age group 35-40 years. The difference was not statistically significant between gestational hypertension and maternal age group. Preeclampsia was $9(7.3 \%)$ in age group $18-29$ years and $2(12.5 \%)$ in age group 35-40 years respectively. Chronic hypertension was $1(6.3 \%)$ in age group 35-40 years. Chronic hypertension was significantly associated with maternal age. However, antepartum hemorrhage, placenta previa, abruptio placenta, gestational hypertension, preeclampsia, eclampsia, GDM, and $\mathrm{BOH}$ were not statistically significant $(\mathrm{p}>0.05)[18]$. Compared with women 30-34 years of age, women aged 45 and older had a statistically significant higher risk of preeclampsia, severe preeclampsia, placenta previa, preterm birth. Lower educational level increased risks for many maternal complications, including preeclampsia, early-onset preeclampsia, severe preeclampsia, GDM (on insulin), placenta previa, and PPH. Compared with patients living in metropolitan, residents of the rural areas had a higher incidence of preeclampsia, preterm birth, low Apgar score, and NICU admission. Patients with insurance were less likely to have preeclampsia and GDM. Regarding malpresentation, breach, transverse lie, congenital malformation, IUD, preterm labour, prolonged labour, obstructed labour and PPH were not statistically significant $(\mathrm{p}>0.05)$. Chinese study revealed a decreasing odds of PPH with increasing age as well [19]. In recent years, the Chinese government has taken action to control the PPH rate [20-21]. In the mode of delivery, normal delivery was found $52(42.3 \%)$ in the age group 18-29 years, 16(39\%) in the age group 30-34 years and $3(18.8 \%)$ in the age group 35-40 years. Caesarean section was $96(58.5 \%)$ and $13(81.3 \%)$ in the age of $<35$ years and $\geq 35$ years respectively. In a study found women aged $\geq 40$ were more likely to have elective CS without medical indications [22]. In another study reported that the CS rate was $66.3 \%$ in women of 35-39 years and $74.8 \%$ in women $\geq 40$ years [23]. Similar to the situation in these studies, some CS was performed following strong maternal request in our hospital. This tendency was further confirmed by our finding of the trend that the influence of primiparity on selective CS was elevated with increasing age. The highest rate of selective $\mathrm{CS}$ and lowest rate of emergency $\mathrm{CS}$ were both in primiparous women $\geq 40$ years. These findings reflected maternal and obstetrician preferences of a low threshold for risk avoidance. In addition, the increased emergency CS rate caused by a higher incidence of having difficulties 
during labour in obese and overweight mothers indicated the importance of weight control in these patients on the other hand. In this study showed low birth weight was found $38(30.9 \%)$ in the age group 18 29 years, $9(22 \%)$ in the age group 30-34 years, and $3(18.8 \%)$ in the age group 35-40 years. IUGR was found $3(2.4 \%)$, stillbirth $6(4.9 \%)$, prematurity $4(3.3 \%)$ and macrosoma $1(0.8 \%)$ in the age group 18-29 years but due to limited sample size only low birth weight was found $3(18.8 \%)$ in age group 35-40 years. NICU admission was needed $36(29.3 \%)$ in the age group of $18-29$ years, $8(19.5 \%)$ in the age group 30-34 years, and $2(12.5 \%)$ in the age group $35-40$ years. The difference was not statistically significant ( $p>0.05)$. Once again, women aged 40 years and older had the highest RR and were almost twice as likely to have a stillborn infant compared to the younger reference population. Adjustment for BMI, parity, ethnic origin, and social deprivation score appeared to increase the RR of stillbirth in the advanced age groups; however, most of the confounding effect was related to adjustment for social deprivation score. The risk of stillbirth was higher for maternal ages 35-39 years but no statistically significant difference was observed for pregnant women aged 40 years or older relative to 20 to 34 year old pregnant women.

\section{CONCLUSION}

In our findings demonstrate that though the fact that being pregnant at any reproductive age is not risk-free for pregnant women, but the reality is still over 35 years are at elevated risk for rising complications and should, therefore, be managed with proper care protocols, particularly in cases of maternal complications of pregnancy. Follow-up data on the health of these women and their babies later in life need to be carefully recorded.

\section{REFERENCE}

1. Frederiksen, L. E., Ernst, A., Brix, N., Lauridsen, L. L. B., Roos, L., Ramlau-Hansen, C. H., \& Ekelund, C. K. (2018). Risk of adverse pregnancy outcomes at advanced maternal age. Obstetrics \& Gynecology, 131(3), 457-463.

2. Wu, Y., Chen, Y., Shen, M., Guo, Y., Wen, S. W., Lanes, A., \& Hua, X. (2019). Adverse maternal and neonatal outcomes among singleton pregnancies in women of very advanced maternal age: a retrospective cohort study. BMC pregnancy and childbirth, 19(1), 3.

3. Care, A. S., Bourque, S. L., Morton, J. S., Hjartarson, E. P., \& Davidge, S. T. (2015). Effect of advanced maternal age on pregnancy outcomes and vascular function in the rat. Hypertension, 65(6), 1324-1330.

4. Hamilton, B. E., Martin, J. A., Osterman, M. J., Curtin, S. C., \& Mathews, T. J. (2015). Births: final data for 2014.
5. Ministry of Health Labor and Welfare. (2013). Vital statistics, Statistics andInformation Department, Ministry of Health, Labor and Welfare, Japan Available at http://www.mhlw.go.jp/toukei/saikin/hw/jinkou/kak utei12/

6. Kenny, L. C., Lavender, T., McNamee, R., O’Neill, S. M., Mills, T., \& Khashan, A. S. (2013). Advanced maternal age and adverse pregnancy outcome: evidence from a large contemporary cohort. PloS one, 8(2).

7. Khalil, A., Syngelaki, A., Maiz, N., Zinevich, Y., \& Nicolaides, K. H. (2013). Maternal age and adverse pregnancy outcome: a cohort study. Ultrasound in Obstetrics \& Gynecology, 42(6), 634-643.

8. Laopaiboon, M., Lumbiganon, P., Intarut, N., Mori, R., Ganchimeg, T., Vogel, J. P., ... \& WHO Multicountry Survey on Maternal Newborn Health Research Network. (2014). Advanced maternal age and pregnancy outcomes: a multicountry assessment. BJOG: An International Journal of Obstetrics \& Gynaecology, 121, 49-56.

9. Ludford, I., Scheil, W., Tucker, G., \& Grivell, R. (2012). Pregnancy outcomes for nulliparous women of advanced maternal age in $\mathrm{S}$ outh A ustralia, 1998-2008. Australian and New Zealand Journal of Obstetrics and Gynaecology, 52(3), 235-241.

10. Waldenström, U., Cnattingius, S., Vixner, L., \& Norman, M. (2017). Advanced maternal age increases the risk of very preterm birth, irrespective of parity: a population-based register study. BJOG: An International Journal of Obstetrics \& Gynaecology, 124(8), 1235-1244.

11. Balasch, J., \& Gratacós, E. (2012). Delayed childbearing: effects on fertility and the outcome of pregnancy. Current Opinion in Obstetrics and Gynecology, 24(3), 187-193.

12. Nilsen, A. B. V., Waldenström, U., Rasmussen, S., Hjelmstedt, A., \& Schytt, E. (2012). O499 characteristics of women who are pregnant with their first baby at an advanced age. International journal of gynecology \& obstetrics, 119, S438S438.

13. Balasch, J., \& Gratacós, E. (2011). Delayed childbearing: effects on fertility and the outcome of pregnancy. Fetal diagnosis and therapy, 29(4), 263-273.

14. Kanungo, J., James, A., McMillan, D., Lodha, A., Faucher, D., Lee, S. K., \& Shah, P. S. (2011). Advanced maternal age and the outcomes of preterm neonates: a social paradox?. Obstetrics \& Gynecology, 118(4), 872-877.

15. Timofeev, J., Reddy, U. M., Huang, C. C., Driggers, R. W., Landy, H. J., \& Laughon, S. K. (2013). Obstetric complications, neonatal morbidity, and indications for cesarean delivery by maternal age. Obstetrics and gynecology, 122(6), 1184.

16. Kenny, L. C., Lavender, T., McNamee, R., O’Neill, S. M., Mills, T., \& Khashan, A. S. (2013). 
Advanced maternal age and adverse pregnancy outcome: evidence from a large contemporary cohort. PloS one, 8(2).

17. Shan, D., Qiu, P. Y., Wu, Y. X., Chen, Q., Li, A. L., Ramadoss, S., ... \& Hu, Y. Y. (2018). Pregnancy outcomes in women of advanced maternal age: a retrospective cohort study from China. Scientific reports, 8(1), 1-9.

18. Ogawa, K., Urayama, K. Y., Tanigaki, S., Sago, H., Sato, S., Saito, S., \& Morisaki, N. (2017). Association between very advanced maternal age and adverse pregnancy outcomes: a cross sectional Japanese study. BMC pregnancy and childbirth, 17(1), 349.

19. Lao TT, Sahota DS, Cheng YK, Law LW, Leung TY. Advanced maternal age and postpartum hemorrhage-risk factor or red herring?. The Journal of Maternal-Fetal \& Neonatal Medicine. 2014 Feb 1;27(3):243-6.

20. National Bureau of Statistcs of China. (2015). China Statistical Yearbook (China Statistics Press, 2015)

21. National Bureu of Statistics of China.(2016). China Statistical Yearbook (China Statistics Press 2016)

22. Ecker, J. L., Chen, K. T., Cohen, A. P., Riley, L. E., \& Lieberman, E. S. (2001). Increased risk of cesarean delivery with advancing maternal age: indications and associated factors in nulliparous women. American journal of obstetrics and gynecology, 185(4), 883-887.

23. Wang, C., Wang, X. Y., \& Yang, H. X. (2017). Effect of maternal age on pregnancy outcomes in Beijing. Zhonghua fu chan ke za zhi, 52(8), 514520. 